IJMMS 25:9 (2001) 587-602

PII. S0161171201004860

http://ijmms.hindawi.com

(C) Hindawi Publishing Corp.

\title{
EVOLUTIONARY EQUATION OF INERTIAL WAVES IN 3-D MULTIPLY CONNECTED DOMAIN WITH DIRICHLET BOUNDARY CONDITION
}

\author{
PAVEL A. KRUTITSKII
}

(Received 23 March 2000)

\begin{abstract}
We study initial-boundary value problem for an equation of composite type in 3-D multiply connected domain. This equation governs nonsteady inertial waves in rotating fluids. The solution of the problem is obtained in the form of dynamic potentials, which density obeys the uniquely solvable integral equation. Thereby the existence theorem is proved. Besides, the uniqueness of the solution is studied. All results hold for interior domains and for exterior domains with appropriate conditions at infinity.
\end{abstract}

2000 Mathematics Subject Classification. Primary 35G15, 35C15, 31B20.

Modern advances in the theory of waves are mostly concerned with nonlinear phenomena $[4,3,5,6]$. However, there exist some types of linear waves which are not well studied yet, for example, nonsteady inertial waves, that is, internal waves in rotating fluids. These waves are governed by PDEs of composite type and of high order. Such PDEs possess both elliptic and hyperbolic characteristics, and so they share properties of both elliptic and hyperbolic equations [13, 23, 24, 25, 26]. Equations of composite type are not well studied even in linear case and they are not covered by existing classifications of PDEs. In particular, they do not belong to equations of principal part, though elliptic, parabolic, and hyperbolic equations belong [7].

Inertial wave equation (see (1.2)) is an evolutionary equation of fourth order and composite type. This equation comes from ocean dynamics. Different initial-boundary value problems for analogous equations were treated in $[8,9,10,14,15,16,17,18$, 19, 20, 30]. In particular, 2-D problems in multiply connected domains were studied in $[15,16,17,18,19,20]$. Initial boundary value problems in simply connected domains were studied for 3-D equation of inertial waves and for similar equations in $[8,9,10,14,30]$, but 3-D problems were not treated in multiply connected domains before. The aim of the present paper is to consider initial-boundary value problem in 3-D multiply connected domain (interior and exterior) with Dirichlet boundary condition. To prove existence theorem for our problem we use dynamic potentials [14] and the method of boundary integral equations. Uniqueness of a solution is also studied. It should be stressed that the integral equation obtained in the present paper is a uniquely solvable Fredholm equation of the second kind and index zero. This equation can be easily computed by discretization and inversion of a matrix. Initial-boundary value problems for some equations of composite type in a 3-D simply connected domain were reduced to integral equations in $[8,10,14]$. However, the method of reduction to integral equations suggested in these papers for simply connected do- 
main is not suitable for multiply connected domain, since obtained integral equations may not have a solution, while the boundary value problem is solvable. Besides, the method of reduction to the integral equation proposed in the present paper is different from those known before, even in case of exterior simply connected domain.

It is a feature of (1.2) that conditions at infinity are required in the exterior domain for the well-posedness of the initial-boundary value problems. However, these conditions were omitted in [14] together with analysis of uniqueness of a solution, and so exterior problems in [14] are not well-posed.

Another approach to studies of initial-boundary value problems for (1.2) in interior domains has been proposed by Sobolev in $[27,28]$. This approach enables us to prove formal existence theorem in Sobolev spaces, but it does not provide us with the integral representation for a solution, and so we do not obtain the constructive way for finding a numerical solution.

1. Formulation of the problem and uniqueness theorem. In Cartesian coordinates $x=\left(x_{1}, x_{2}, x_{3}\right) \in R^{3}$ we consider the connected domain $\mathscr{D}$ with the boundary $\Gamma$ of class $C^{2, \lambda}, \lambda \in(0,1]$. Suppose that $\Gamma$ consists of simple closed surfaces $\Gamma_{0}, \Gamma_{1}, \ldots, \Gamma_{N}$, without common points, so that $\Gamma_{0}$ envelopes all others. The surface $\Gamma_{0}$ may be absent, then the domain $\mathscr{D}$ is unbounded. By $\mathbf{n}_{x}$ we denote interior normal vector with respect to the domain $\mathscr{D}$ at the point $x \in \Gamma$. Consider surfaces making up $\Gamma$ as double sided. The side of surfaces $\Gamma$, which can be seen if we look towards the normal's tips $\mathbf{n}_{x}(x \in \Gamma)$, is denoted by $\Gamma^{-}$, and the opposite side is denoted by $\Gamma^{+}$.

DEFINITION 1.1. The function $U(t, x)$ defined in $[0, \infty) \times \bar{D}$ belongs to the smoothness class $G$, if

(1) $U, u_{t} \in C^{0}([0, \infty) \times \mathscr{D}) ;\left(\partial^{k} / \partial t^{k}\right)\left(\partial^{p} / \partial x_{j}^{p}\right) u \in C^{0}((0, \infty) \times \mathscr{D}), k, p=0,1,2 ; j=$ $1,2,3$;

(2) for any $t \geq 0$ the limiting value of the function $U$ exists on the boundary $\Gamma$ in the sense of a limit in a normal direction to a boundary.

DEFINITION 1.2. Function $u(t, x)$ defined in an unbounded domain is called regular at infinity if there exist such functions $q_{1}(t), q_{2}(t) \in C^{0}[0, \infty)$, that as $|x| \rightarrow \infty$ the inequalities hold

$$
\left|\frac{\partial^{j}}{\partial t^{j}} u(t, x)\right| \leq q_{1}(t)|x|^{-1}, \quad\left|\frac{\partial^{j}}{\partial t^{j}} u_{x_{k}}(t, x)\right| \leq q_{2}(t)|x|^{-2} \text {, where } j=0,1,2 ; k=1,2,3 \text {. }
$$

Equation of inertial waves in a rotating fluid in the 3-D case has the form [1, 11, 14, 21, 22]:

$$
\frac{\partial^{2}}{\partial t^{2}} \Delta u+\omega^{2} u_{x_{3} x_{3}}=0, \quad \triangle=\frac{\partial^{2}}{\partial x_{1}^{2}}+\frac{\partial^{2}}{\partial x_{2}^{2}}+\frac{\partial^{2}}{\partial x_{3}^{2}},
$$

where $\omega \geq 0$ is twice the angular velocity of a rotation around $O x_{3}$ axis. We assume that $\omega$ is a constant.

Let us formulate the initial-boundary value problem for (1.2) in the domain $\mathscr{D}$ for $t \geq 0$. 
Problem $\mathscr{K}$. To find a function $\mathcal{U}(t, x)$ of class $G$, which obeys $(1.2)$ in $(0, \infty) \times \mathscr{D}$ in a classical sense, that satisfies the initial conditions $U(0, x)=U_{t}(0, x)=0, x \in \mathscr{D}$ and the boundary condition on $\Gamma$ :

$$
\left.u(t, x)\right|_{x \in \Gamma}=\left.f_{0}(t, x)\right|_{x \in \Gamma}, \quad t \geq 0 .
$$

Besides, if $\mathscr{D}$ is an exterior domain, then the function $U(t, x)$ must be regular at infinity.

The boundary condition should be satisfied in a sense of a limit in a normal direction to the boundary $\Gamma$.

Together with the class $G$ we consider class of smoothness $G_{1}$.

DEFINITION 1.3. Function $U(t, x)$ belongs to the class $G_{1}$, if

(1) $u, u_{t} \in C^{0}([0, \infty) \times \bar{D}) ; \nabla \mathcal{U}, \nabla \mathcal{U}_{t} \in C^{0}([0, \infty) \times \mathscr{D})$; $\left(\partial^{k} / \partial t^{k}\right)\left(\partial^{p} / \partial x_{j}^{p}\right) u \in C^{0}((0, \infty) \times \mathscr{D}), k, p=0,1,2 ; j=1,2,3$;

(2) for any $t \geq 0$, there exists the uniform limit, for all $x \in \Gamma$, of the expression

$$
\mathcal{N}_{t x} U(t, \bar{x})=\frac{\partial^{2}}{\partial t^{2}}\left(\mathbf{n}_{x}, \nabla_{\bar{x}}\right) \cup(t, \bar{x})+\omega^{2} \cos \left(\mathbf{n}_{x}, x_{3}\right) u_{x_{3}}(t, \bar{x})
$$

as $\bar{x}$ tends to $x \in \Gamma$ along the normal $\mathbf{n}_{x}$, that is, the limit is understood in the sense of a limit along a normal to a boundary $\Gamma$. The cosine of the angle between the normal vector $\mathbf{n}_{x}$ in the point $x \in \Gamma$ and the direction of the $O x_{3}$ axis is denoted by $\cos \left(\mathbf{n}_{x}, x_{3}\right)$.

NotE. Evidently, any function of class $G_{1}$ belongs to the class $G$. Below $\int_{\Gamma} \cdots d S$ denotes the surface integral of the first kind.

Consider the uniqueness theorem for the problem $\mathscr{K}$.

LEMMA 1.4. Let $U(t, x)$ be a classical solution of $(1.2)$ in $(0, \infty) \times \mathscr{D}$, and $U(t, x)$ belongs to class $G_{1}$. Besides, assume that the function $u(t, x)$ is regular at infinity in case of exterior domain $\mathscr{D}$. Then for any $t \geq 0$ the identity holds

$$
\frac{1}{2} \frac{d}{d t}\left(\left\|\nabla u_{t}\right\|^{2}+\omega^{2}\left\|u_{x_{3}}\right\|^{2}\right)=\int_{\Gamma} u_{t} \mathcal{N}_{t x} u d s
$$

where the norm $\|\cdot\|$ is taken in the space $L_{2}(\mathscr{D})$.

Lemma 1.4 can be proved by direct verification. To do so we construct equidistant surfaces [32] in the domain $\mathscr{D}$ to the boundary $\Gamma$. If $\mathscr{D}$ is an exterior domain, then additionally we consider a sphere of enough large radius $R$ with the center in the origin. In the domain bounded by equidistant surfaces (and the sphere if $\mathscr{D}$ is an exterior domain), we write energy equality, which is obtained as a result of multiplying equation (1.2) by $u_{t}$ and integration by parts. Tending equidistant surfaces to the boundary $\Gamma$ and using properties of functions of class $G_{1}$, we prove the identity stated in Lemma 1.4, in case of interior domain $\mathscr{D}$. If $\mathscr{D}$ is an exterior domain, then additionally we tend $R$ to infinity and use regularity of $u(t, x)$ at infinity. Finally, we arrive at the identity stated in Lemma 1.4 again.

Next theorem follows from Lemma 1.4. 
THEOREM 1.5. If the problem $\mathscr{K}$ has a solution in the smoothness class $G_{1}$, then this solution is unique.

Solvability of the problem $\mathscr{K}$ will be studied under weaker smoothness conditions on its solutions, than in Theorem 1.5. More precisely, we drop the condition that solution belongs to the class $G_{1}$. We will prove classical solvability of the problem $\mathcal{K}$ and derive integral representation for a solution in the form of dynamic potentials (see Theorem 4.2). Density in potentials obeys integral equation, which is solvable.

We now turn to the construction of the classical solution of the problem $\mathscr{K}$.

2. Reduction of the problem to the integral equation. Let us introduce some classes of functions. Set

$$
C_{0}^{2}[0, \infty)=\left\{\varphi(t): \varphi(t) \in C^{2}[0, \infty), \varphi(0)=\varphi^{\prime}(0)=0\right\} .
$$

By $C^{k}([0, \infty) ; \mathscr{H})$ we denote class of $k$ times continuously differentiable at $t \geq 0$ abstract functions $\varphi(t)$ with values in the Banach space $\mathscr{H}$. Besides, we introduce the following class of abstract functions:

$$
C_{0}^{2}([0, \infty) ; \mathscr{H})=\left\{\varphi(t): \varphi(t) \in C^{2}([0, \infty) ; \mathscr{H}), \varphi(0)=\varphi^{\prime}(0)=0\right\} .
$$

By the designation $C^{0}([0, T] ; \mathscr{H})$, where $T>0$, we mean Banach space of abstract functions with the norm

$$
\|\cdot\|_{C^{0}([0, T] ; \mathscr{H})}=\|\| \cdot\left\|_{\mathscr{H}}\right\|_{C^{0}[0, T]} \cdot
$$

Further on, we use the following result from the theory of abstract functions (see proof in [29, pages 28-29]).

LEMmA 2.1. Classes of functions $C^{0}\left([0, \infty) ; C^{0}(\Gamma)\right)$ and $C^{0}([0, \infty) \times \Gamma)$ are equivalent, that is, any function of the first class belongs to the second and conversely.

Let us construct the solution of the problem $\mathscr{K}$. Assume that the function $f_{0}(t, x)$ in the boundary condition (1.3) belongs to the class $C_{0}^{2}\left([0, \infty) ; C^{0}(\Gamma)\right)$, then it can be expressed in the form

$$
f_{0}(t, x)=\int_{0}^{t} \int_{0}^{t_{1}} f\left(t_{2}, x\right) d t_{2} d t_{1}=\int_{0}^{t}(t-\tau) f(\tau, x) d \tau,
$$

where

$$
f(t, x)=\frac{\partial^{2}}{\partial t^{2}} f_{0}(t, x) \in C^{0}\left([0, \infty) ; C^{0}(\Gamma)\right) .
$$

By $J_{0}(\sigma)$ and $J_{1}(\sigma)$ we denote Bessel functions of zero and first order.

Consider a function introduced in [14] and called dynamic double layer potential for (1.2),

$$
\begin{aligned}
U[\bar{\mu}](t, x)= & \int_{0}^{t} \int_{\Gamma} \bar{\mu}(\tau, y) \mathcal{N}_{\tau} W(t-\tau, x-y) d S_{y} d \tau \\
& -\frac{1}{4 \pi} \int_{\Gamma} \bar{\mu}(t, y) \frac{\partial}{\partial \mathbf{n}_{y}} \frac{1}{|x-y|} d S_{y}
\end{aligned}
$$


where $x=\left(x_{1}, x_{2}, x_{3}\right) \in \mathscr{D}, y=\left(y_{1}, y_{2}, y_{3}\right) \in \Gamma$, operator $\mathcal{N}_{t x}$ was introduced in (1.4),

$$
W(t, x)=-\frac{1}{4 \pi|x|_{0}} \int_{0}^{t|x|_{0} /|x|} J_{0}(\sigma) d \sigma=-\frac{t}{4 \pi|x|}+\frac{|x|_{0}}{4 \pi|x|^{2}}\left(t * J_{1}\left(t \frac{|x|_{0}}{|x|}\right)\right)
$$

is a singular solution for (1.2), $|x|=\left(x_{1}^{2}+x_{2}^{2}+x_{3}^{2}\right)^{1 / 2},|x|_{0}=\omega\left(x_{1}^{2}+x_{2}^{2}\right)^{1 / 2}$. By the symbol $*$ the convolution operation in time is denoted so that for any two functions of time $a(t), b(t)$, we have

$$
a(t) * b(t)=\int_{0}^{t} a(t-\tau) b(\tau) d \tau=\int_{0}^{t} a(\tau) b(t-\tau) d \tau .
$$

With respect to the density $\bar{\mu}(t, x)$ we assume that it has the form

$$
\bar{\mu}(t, x)=\int_{0}^{t} \int_{0}^{t_{1}} \mu\left(t_{2}, x\right) d t_{2} d t_{1}=\int_{0}^{t}(t-\tau) \mu(\tau, x) d \tau,
$$

where $\mu(t, x) \in C^{0}\left([0, \infty) ; C^{0}(\Gamma)\right)$, so that $\bar{\mu}(t, x) \in C_{0}^{2}\left([0, \infty) ; C^{0}(\Gamma)\right)$.

As shown in [14], the function $W(t, x)$ for $x \neq 0$ obeys (1.2) in a classical sense, and $W(0, x)=0, W_{t}(0, x)=-(4 \pi|x|)^{-1}$.

For the function $\mathcal{N}_{\tau y} W(t-\tau, x-y)$ the following representation holds [14]:

$$
\begin{aligned}
& \mathcal{N}_{\tau y} W(t-\tau, x-y) \\
& \quad=\frac{\cos \left(x-y, \mathbf{n}_{y}\right)}{4 \pi|x-y|^{2}}\left[\frac{|x-y|_{0}}{|x-y|} J_{0}^{\prime}(\zeta)+\frac{(\tau-t)\left(x_{3}-y_{3}\right)^{2}}{|x-y|^{2}} \omega^{2} J_{0}(\zeta)\right], \quad \zeta=\frac{(\tau-t)|x-y|_{0}}{|x-y|} .
\end{aligned}
$$

Here $\cos \left(x-y, \mathbf{n}_{y}\right)$ is a cosine of the angle between the vectors $\overrightarrow{y x}$ and $\mathbf{n}_{y}$. The function in the square brackets is continuous for $x \neq y$ and uniformly bounded in all variables on any finite interval of time. Since $\left|\cos \left(x-y, \mathbf{n}_{y}\right)\right| \leq \operatorname{const}|x-y|^{\lambda_{1}}$ for $x, y \in \Gamma$ and $\Gamma \in C^{1, \lambda_{1}}$ (recall that in our case $\Gamma \in C^{2, \lambda}$ ), then as shown in [14], the potential $U[\bar{\mu}](t, x)$ is continuous for $x \in \Gamma$.

Next lemma follows from the properties of double layer dynamic potential, presented in [14].

LEMMA 2.2. If $\mu(t, s) \in C^{0}\left([0, \infty) ; C^{0}(\Gamma)\right)$ and $\bar{\mu}(t, s)$ is given by (2.9), then the potential $U[\bar{\mu}](t, x)$ belongs to the class $G$, obeys $(1.2)$ in $(0, \infty) \times \mathscr{D}$ and initial conditions of the problem $\mathscr{K}$. Besides, if $\mathscr{D}$ is exterior domain, then the potential $U[\bar{\mu}](t, x)$ is regular at infinity.

By $Y_{1}, \ldots, Y_{N}$ we denote an arbitrary fixed points placed inside surfaces $\Gamma_{1}, \ldots, \Gamma_{N}$ and consider a function

$$
\begin{aligned}
Q[\bar{\mu}](t, x)= & \sum_{k=1}^{N} \int_{0}^{t} \int_{\Gamma_{k}} \mu(\tau, y) d S_{y} W\left(t-\tau, x-Y_{k}\right) d \tau \\
=\frac{1}{4 \pi} \sum_{k=1}^{N}\{ & -\frac{1}{\left|x-Y_{k}\right|} \int_{\Gamma_{k}} \bar{\mu}(t, y) d S_{y}+\frac{\left|x-Y_{k}\right|_{0}}{\left|x-Y_{k}\right|^{2}} \\
& \left.\times \int_{0}^{t} \int_{\Gamma_{k}} \bar{\mu}(\tau, y) d S_{y} J_{1}\left(\frac{\left|x-Y_{k}\right|_{0}}{\left|x-Y_{k}\right|}(t-\tau)\right) d \tau\right\},
\end{aligned}
$$

where $W(t, x)$ was defined above. 
Function $Q[\bar{\mu}](t, x)$ is a linear combination of point sources for (1.2) placed in the points $Y_{k}, k=1, \ldots, N$. If conditions of Lemma 2.2 hold, then $Q[\bar{\mu}](t, x)$ belongs to the class $G$, obeys $(1.2)$ in $(0, \infty) \times \mathscr{D}$ and satisfies initial conditions of the problem $\mathscr{K}$. Moreover, $Q[\bar{\mu}](t, x)$ is infinitely differentiable in $x$ in $R^{3} \backslash\left(\bigcup_{k=1}^{N} Y_{k}\right)$ and is regular at infinity.

The solution of the problem $\mathscr{K}$ we seek in the form

$$
u[\bar{\mu}](t, x)=U[\bar{\mu}](t, x)+Q[\bar{\mu}](t, x),
$$

where $\bar{\mu}(t, x)$ is defined by (2.9), for $U[\bar{\mu}](t, x)$ the representation (2.6) holds, and $Q[\bar{\mu}](t, x)$ is given by expression (2.11). If we drop the function $Q[\bar{\mu}](t, x)$ in (2.12), then the integral equation obtained below may be not solvable.

The above properties of functions occurring in (2.12) yield the following assertion.

LEMMA 2.3. If conditions of Lemma 2.2 hold, then the function (2.12) belongs to the class $G$, satisfies $(1.2)$ in $(0, \infty) \times \mathscr{D}$ and initial conditions of the problem $\mathcal{K}$. In addition, if $\mathscr{D}$ is an exterior domain, then the function (2.12) is regular at infinity.

Thus, $\mathcal{U}[\bar{\mu}](t, x)$ satisfies all conditions of the problem $\mathscr{K}$, except for the boundary condition (1.3). Using formulas for limiting values of double layer dynamic potential at the boundary of a domain [14] and satisfying the boundary condition (1.3), we arrive at integral equation with respect to the function $\mu(t, x)$,

$$
-\frac{1}{2} \mu(t, x)-(A \mu)(t, x)+(B \mu)(t, x)=f(t, x), \quad x \in \Gamma,
$$

where $f(t, x)=\left(f_{0}(t, x)\right)_{t t} \in C^{0}\left([0, \infty) ; C^{0}(\Gamma)\right), f_{0}(t, x) \in C_{0}^{2}\left([0, \infty) ; C^{0}(\Gamma)\right)$. By $A$ and $B$ the following operators are denoted:

$$
\begin{aligned}
(A \mu)(t, x) & =\int_{\Gamma} \mu(t, y) \mathscr{A}(x, y) d S_{y}, \\
(B \mu)(t, x) & =\int_{0}^{t} \int_{\Gamma} \mu(\tau, y) \mathscr{B}(t-\tau, x, y) d S_{y} d \tau, \\
\mathscr{B}(t-\tau, x, y) & =\mathcal{N}_{\tau y} W(t-\tau, x-y)+\frac{1}{4 \pi} \sum_{k=1}^{N} \delta_{k}(y) \frac{\left|x-Y_{k}\right|_{0}}{\left|x-Y_{k}\right|^{2}} J_{1}\left((t-\tau) \frac{\left|x-Y_{k}\right|_{0}}{\left|x-Y_{k}\right|}\right), \\
\mathscr{A}(x, y) & =\frac{1}{4 \pi} \frac{\partial}{\partial \mathbf{n}_{y}} \frac{1}{|x-y|}+\frac{1}{4 \pi} \sum_{k=1}^{N} \delta_{k}(y) \frac{1}{\left|x-Y_{k}\right|} .
\end{aligned}
$$

Here $\mathcal{N}_{\tau y} W(t-\tau, x-y)$ is a function from (2.10) and

$$
\delta_{k}(y)= \begin{cases}1, & y \in \Gamma_{k}, \\ 0, & y \notin \Gamma_{k} .\end{cases}
$$

Let us study some properties of operators $A$ and $B$. 
LEMMA 2.4. (1) Kernels $\mathscr{A}(x, y), \mathscr{B}(t, x, y)$ are continuous for $x \neq y$ and have $a$ weak singularity if $x=y$.

(2) The operator $A$ acts from $C^{0}(\Gamma)$ into $C^{0}(\Gamma)$ and bounded.

(3) The operator $B$ acts from $C^{0}\left([0, \infty) ; C^{0}(\Gamma)\right)$ into $C^{0}\left([0, \infty) ; C^{0}(\Gamma)\right)$.

(4) If $h(t, x) \in C^{0}\left([0, \infty) ; C^{0}(\Gamma)\right)$, then for any $t \geq 0$ the following inequality holds:

$$
\|(B h)(t, \cdot)\|_{C^{0}(\Gamma)} \leq c_{B}\left[\int_{0}^{t}\|h(\tau, \cdot)\|_{C^{0}(\Gamma)} d \tau+\int_{0}^{t} \int_{0}^{t_{1}}\|h(\tau, \cdot)\|_{C^{0}(\Gamma)} d \tau d t_{1}\right],
$$

where $c_{B}$ is a constant.

Proof. Item (1) can be verified directly. Kernel $\mathscr{A}(x, y)$ has been studied in [32]. Properties of kernel $\mathscr{B}(t, x, y)$ follow from the representation (2.10).

Item (2) follows from [32, Section 17.4] and item (1), since the kernel of the operator $A$ is polar.

For item (3), let $h(t, x) \in C^{0}\left([0, \infty) ; C^{0}(\Gamma)\right)$. According to Lemma 2.1, $h(t, x) \in$ $C^{0}([0, \infty) \times \Gamma)$. Set

$$
\begin{aligned}
& (B h)(t, x)=(\hat{B} h)(t, x)+(\check{B} h)(t, x), \\
& (\hat{B} h)(t, x)=\int_{0}^{t} \int_{\Gamma} h(\tau, y) \mathcal{N}_{\tau y} W(t-\tau, x-y) d S_{y} d \tau, \\
& (\check{B} h)(t, x)=\int_{0}^{t} \int_{\Gamma} h(\tau, y)\left(\mathscr{B}(t-\tau, x, y)-\mathcal{N}_{\tau y} W(t-\tau, x-y)\right) d S_{y} d \tau .
\end{aligned}
$$

It follows from the results of [14] that the operator $\hat{B}$ acts from $C^{0}\left([0, \infty) ; C^{0}(\Gamma)\right)$ into $C^{0}\left([0, \infty) ; C^{0}(\Gamma)\right)$. From the explicit expression for the kernel $(\mathscr{B}(t-\tau, x, y)-$ $\left.\mathcal{N}_{\text {T } y} W(t-\tau, x-y)\right)$ it follows that this kernel is continuous. Hence the operator $\breve{B}$ acts from $C^{0}([0, \infty) \times \Gamma)$ into $C^{0}([0, \infty) \times \Gamma)$. Taking into account Lemma 2.1, we are convinced that the operator $\breve{B}$ acts from $C^{0}\left([0, \infty) ; C^{0}(\Gamma)\right)$ into $C^{0}\left([0, \infty) ; C^{0}(\Gamma)\right)$. Consequently, item (3) of lemma holds for operator $B=\hat{B}+\breve{B}$.

Let us prove (4). Let $\Gamma \in C^{1, \lambda_{1}} \subset C^{2, \lambda}, \lambda_{1} \in(0,1]$. If $x, y \in \Gamma$, then the following estimates hold:

$$
\begin{gathered}
\left|\mathcal{N}_{\tau y} W(t-\tau, x-y)\right| \leq c_{1} \frac{\left|\cos \left(x-y, \mathbf{n}_{y}\right)\right|}{|x-y|^{2}}[1+(t-\tau)], \\
\mathscr{B}(t-\tau, x, y) \leq c_{1} \frac{\left|\cos \left(x-y, \mathbf{n}_{y}\right)\right|}{|x-y|^{2}}[1+(t-\tau)]+c_{2},
\end{gathered}
$$

where $c_{1}, c_{2}$ are some constants and first estimate follows from (2.10).

Let $h(t, x) \in C^{0}\left([0, \infty) ; C^{0}(\Gamma)\right)$, then for operator $B$ the following estimate holds:

$$
\begin{aligned}
|(B h)(t, x)| & \leq \int_{0}^{t} \int_{\Gamma}|h(\tau, y)|\left\{c_{1} \frac{\left|\cos \left(x-y, \mathbf{n}_{y}\right)\right|}{|x-y|^{2}}[1+(t-\tau)]+c_{2}\right\} d S_{y} d \tau \\
& \leq \int_{0}^{t}\|h(\tau, \cdot)\|_{C^{0}(\Gamma)}\left\{c_{1} \int_{\Gamma} \frac{\left|\cos \left(x-y, \mathbf{n}_{y}\right)\right|}{|x-y|^{2}} d S_{y}[1+(t-\tau)]+c_{2} \int_{\Gamma} 1 d S_{y}\right\} d \tau \\
& \leq \int_{0}^{t}\|h(\tau, \cdot)\|_{C^{0}(\Gamma)}\left\{\tilde{c}_{1}[1+(t-\tau)]+\tilde{c}_{2}\right\} d \tau \\
& =\tilde{c}_{1} \int_{0}^{t} \int_{0}^{t_{1}}\|h(\tau, \cdot)\|_{C^{0}(\Gamma)} d \tau d t_{1}+\left(\tilde{c}_{1}+\tilde{c}_{2}\right) \int_{0}^{t}\|h(\tau, \cdot)\|_{C^{0}(\Gamma)} d \tau,
\end{aligned}
$$


where $\tilde{c}_{1}, \tilde{c}_{2}$ are some constants and Lemma 2.1 from [32, Section 27.4] has been used. This lemma states that the integral

$$
\int_{\Gamma} \frac{\left|\cos \left(x-y, \mathbf{n}_{y}\right)\right|}{|x-y|^{2}} d S_{y}
$$

is uniformly bounded by a constant for all $x \in \Gamma$. Taking $\sup _{x \in \Gamma}$ on both sides of the inequality (2.19), we obtain

$$
\|(B h)(t, \cdot)\|_{C^{0}(\Gamma)} \leq\left(\tilde{c}_{1}+\tilde{c}_{2}\right)\left\{\int_{0}^{t} \int_{0}^{t_{1}}\|h(\tau, \cdot)\|_{C^{0}(\Gamma)} d \tau d t_{1}+\int_{0}^{t}\|h(\tau, \cdot)\|_{C^{0}(\Gamma)} d \tau\right\},
$$

and so we complete the proof of estimate (2.16).

In the next section, we continue analysis of operators included in integral equation (2.13).

3. Properties of operators included in the integral equation. On the contour $\Gamma$ we consider integral equation with respect to the function $h(x) \in C^{0}(\Gamma)$,

$$
-\frac{1}{2} h(x)-(A h)(x)=g(x), \quad x \in \Gamma,
$$

where $A$ is an operator from (2.13), and $g(x)$ belongs to $C^{0}(\Gamma)$. According to Lemma 2.4, the kernel of the operator $A$ is polar, therefore (3.1) is a Fredholm equation of the second kind and index zero.

Let us prove that (3.1) is uniquely solvable in $C^{0}(\Gamma)$. To this end, it is sufficient to show that homogeneous equation (3.1) has only a trivial solution. We give a proof by contradiction. Let the homogeneous equation have a nontrivial solution $h_{0}(x) \in C^{0}(\Gamma)$ for which the identity holds

$$
-\frac{1}{2} h_{0}(x)-\left(A h_{0}\right)(x)=0, \quad x \in \Gamma .
$$

We write this identity in the form

$$
-\frac{1}{2} h_{0}(x)=\int_{\Gamma} h_{0}(y) \mathscr{A}_{1}(x, y) d S_{y}+\int_{\Gamma} h_{0}(y) \mathscr{A}_{2}(x, y) d S_{y},
$$

where

$$
\mathscr{A}_{1}(x, y)=\frac{1}{4 \pi} \frac{\partial}{\partial \mathbf{n}_{y}} \frac{1}{|x-y|}, \quad \mathscr{A}_{2}(x, y)=\frac{1}{4 \pi} \sum_{k=1}^{N} \delta_{k}(y) \frac{1}{\left|x-Y_{k}\right|} .
$$

We show that $h_{0}(x) \in C^{1, \omega}(\Gamma)$ with $\omega \in(0,1)$. Indeed, the second integral term in the right part of (3.3) is infinitely differentiable in $x$ since it does not have any singularity at $x=y$. According to [2, Theorem 2.15], the first integral term in the right-hand side of (3.3) is a Hölder function in $x$ on $\Gamma$. It follows from the identity (3.3) for the function $h_{0}(x)$ that $h_{0}(x)$ is also Hölder function in $x$ on $\Gamma$. Using [2, Theorem 2.22], we obtain that the first integral term in (3.3) belongs to $C^{1, \omega}(\Gamma)$ in $x$ for some $\omega \in(0,1)$. Now from the identity (3.3) for the function $h_{0}(x)$ we get: $h_{0}(x) \in C^{1, \omega}(\Gamma), \omega \in(0,1)$. So, we complete the proof. 
LEMMA 3.1. Any solution of the homogeneous equation (3.1) (with $g \equiv 0$ ) in $C^{0}(\Gamma)$ automatically belongs to $C^{1, \omega}(\Gamma)$, where $\omega \in(0,1)$.

Consider a harmonic function in $\mathscr{D}$ constructed with the help of $h_{0}(x)$ in the form of a sum of double layer potential and point sources placed inside surfaces $\Gamma_{k}$ in the points $Y_{k}$,

$$
v\left[h_{0}\right](x)=-\frac{1}{4 \pi} \int_{\Gamma} h_{0}(y) \frac{\partial}{\partial \mathbf{n}_{y}} \frac{1}{|x-y|} d S_{y}-\frac{1}{4 \pi} \sum_{k=1}^{N} \int_{\Gamma_{k}} h_{0}(y) d S_{y} \frac{1}{\left|x-Y_{k}\right|} .
$$

This function belongs to $C^{0}(\bar{D}) \cap C^{2}(\mathscr{D})$ and meets all conditions of the homogeneous Dirichlet problem

$$
\triangle v(x)=0, \quad x \in \mathscr{D} ;\left.\quad v\right|_{\Gamma}=0 .
$$

Besides, if $\mathscr{D}$ is an exterior domain, then

$$
|v(x)|=O\left(|x|^{-1}\right), \quad \text { as }|x| \longrightarrow \infty .
$$

Note that when satisfying the boundary condition $\left.v\right|_{\Gamma}=0$, we obtain identity (3.3). According to the maximum principle for harmonic functions, the solution of this Dirichlet problem is unique. Consequently,

$$
v\left[h_{0}\right](x) \equiv 0, \quad x \in \bar{D} .
$$

By $\mathscr{D}_{j}$ we denote the interior simply connected domain, bounded by the surface $\Gamma_{j}(j=1, \ldots, N)$. Let $\mathscr{f}$ be simple smooth closed surface, lying in the domain $\mathscr{D}$ and enveloping only the surface $\Gamma_{j}$ among all surfaces making up $\Gamma$. Assuming that $\mathbf{n}_{x}$ is continuous normal on the surface $\mathscr{f}$, we compute

$$
\begin{aligned}
\int_{\mathscr{g}} \frac{\partial v\left[h_{0}\right](x)}{\partial \mathbf{n}_{x}} d S_{x}=0= & -\int_{\Gamma_{j}} h_{0}(y) \frac{\partial}{\partial \mathbf{n}_{y}}\left(\frac{1}{4 \pi} \int_{\mathscr{g}} \frac{\partial}{\partial \mathbf{n}_{x}} \frac{1}{|x-y|} d S_{x}\right) d S_{y} \\
& -\int_{\Gamma_{j}} h_{0}(y) d S_{y} \frac{1}{4 \pi} \int_{\mathscr{g}} \frac{\partial}{\partial \mathbf{n}_{x}} \frac{1}{\left|x-Y_{j}\right|} d S_{x} .
\end{aligned}
$$

Here we take into account the following property of harmonic functions: if the function $V(x)$ is harmonic inside the surface $\mathscr{F}$ and $\nabla V(x)$ can be continuously extended on $\mathscr{F}$ from inside, then

$$
\int_{\mathscr{g}} \frac{\partial V}{\partial \mathbf{n}_{x}} d S_{x}=0 .
$$

We keep in mind that thanks to the Ostrogradsky-Gauss formula

$$
\frac{1}{4 \pi} \int_{\mathscr{y}} \frac{\partial}{\partial \mathbf{n}_{x}} \frac{1}{|x-y|} d S_{x}=\frac{1}{4 \pi} \int_{\mathscr{y}} \frac{\partial}{\partial \mathbf{n}_{x}} \frac{1}{\left|x-Y_{j}\right|} d S_{x}= \pm 1
$$

where the sign depends on the selection of the direction of the normal $\mathbf{n}_{x}$ on the surface $g$. Hence,

$$
\int_{\mathscr{g}} \frac{\partial v\left[h_{0}\right](x)}{\partial \mathbf{n}_{x}} d S_{x}=\mp \int_{\Gamma_{j}} h_{0}(y) d S_{y}=0 .
$$


So, we obtain the identity

$$
\int_{\Gamma_{j}} h_{0}(y) d S_{y}=0
$$

which guarantees that the function $v\left[h_{0}\right](x)$ does not have a point source inside the surface $\Gamma_{j}$. Therefore $v\left[h_{0}\right](x)$ is a harmonic function in $\mathscr{D}_{j}$. Proceeding from Lemma 3.1, $h_{0}(x) \in C^{1, \omega}(\Gamma)$. As shown in [2, Theorem 2.23] under such density $h_{0}(x)$ the derivatives of the double layer potential in $v\left[h_{0}\right](x)$ are continuously extended on $\Gamma_{j}$ from inside and outside. Moreover,

$$
\lim _{\substack{x^{1} \rightarrow x \in \Gamma_{j} \\ x^{1} \in \mathscr{I}}} \frac{\partial v\left[h_{0}\right]\left(x^{1}\right)}{\partial \mathbf{n}_{x}}=\lim _{\substack{x^{1} \rightarrow x \in \Gamma_{j} \\ x^{1} \in \mathscr{I}_{j}}} \frac{\partial v\left[h_{0}\right]\left(x^{1}\right)}{\partial \mathbf{n}_{x}}=0,
$$

where (3.8) has been used. Clearly, the function $v\left[h_{0}\right](x)$ satisfies the following Neumann problem in $\mathscr{D}_{j}$ :

$$
\Delta v(x)=0, \quad x \in \mathscr{D}_{j} ;\left.\quad \frac{\partial v}{\partial \mathbf{n}_{x}}\right|_{\Gamma_{j}}=0 .
$$

It is well known [32] that the solution of such problem is a constant, that is,

$$
v\left[h_{0}\right](x) \equiv \text { const }=c, \quad x \in \mathscr{D}_{j} .
$$

Employing the theorem on the jump of the double layer potential and using (3.8), we obtain

$$
\lim _{\substack{x^{1} \rightarrow x \in \Gamma_{j} \\ x^{1} \in \mathscr{D}}} v\left[h_{0}\right]\left(x^{1}\right)-\lim _{\substack{x^{1} \rightarrow x \in \Gamma_{j} \\ x \in \mathscr{I}_{j}}} v\left[h_{0}\right]\left(x^{1}\right)=-h_{0}(x)=-c,
$$

so that $h_{0}(x)=c$ on $\Gamma_{j}$. It follows from (3.13), that $c=0$. Therefore $h_{0}(x) \equiv 0$ on $\Gamma_{j}$. Recall that $\Gamma_{j}$ is an arbitrary closed surface $(j=1, \ldots, N)$, belonging to $\Gamma$. Hence, $h_{0}(x) \equiv 0$ on $\Gamma_{1}, \ldots, \Gamma_{N}$. Thus, $h_{0}(x) \equiv 0$ on $\Gamma$ if $\mathscr{D}$ is an exterior domain and $h_{0}(x) \equiv 0$ on $\Gamma \backslash \Gamma_{0}$ if $\mathscr{D}$ is an interior domain. Finally, we should clarify the value of $h_{0}(x)$ on $\Gamma_{0}$ in case of interior domain $\mathscr{D}$.

Let $\mathscr{D}$ be an interior domain, then $h_{0}(x) \equiv 0$ on $\Gamma \backslash \Gamma_{0}$, and the function $v\left[h_{0}\right](x)$ take the form of double layer potential

$$
v\left[h_{0}\right](x)=-\frac{1}{4 \pi} \int_{\Gamma_{0}} h_{0}(y) \frac{\partial}{\partial \mathbf{n}_{y}} \frac{1}{|x-y|} d S_{y} .
$$

Let $\mathscr{D}_{0}$ be an exterior domain, bounded by the surface $\Gamma_{0}$. The potential $v\left[h_{0}\right](x)$ is harmonic in $\mathscr{D}_{0}$ and $v(x)=O\left(|x|^{-1}\right), \nabla v(x)=O\left(|x|^{-2}\right)$ as $|x| \rightarrow \infty$. Taking into account smoothness of $h_{0}(x)$ on $\Gamma$, ensured by the Lemma 3.1 and employing [22, Theorem 2.23], we obtain

$$
\lim _{\substack{x^{1} \rightarrow x \in \Gamma_{0} \\ x^{1} \in \mathscr{D}}} \frac{\partial v\left[h_{0}\right]\left(x^{1}\right)}{\partial \mathbf{n}_{x}}=\lim _{\substack{x^{1} \rightarrow x \in \Gamma_{0} \\ x^{1} \in \mathscr{I}_{0}}} \frac{\partial v\left[h_{0}\right]\left(x^{1}\right)}{\partial \mathbf{n}_{x}}=0,
$$


where (3.8) has been used. Consequently, the function $v\left[h_{0}\right](x)$ satisfies the following Neumann problem in $\mathscr{D}_{0}$ :

$$
\begin{gathered}
\Delta v(x)=0, \quad x \in \mathscr{D}_{0} ;\left.\quad \frac{\partial v}{\partial \mathbf{n}_{x}}\right|_{\Gamma_{0}}=0 ; \\
v(x)=O\left(|x|^{-1}\right), \quad \nabla v(x)=O\left(|x|^{-2}\right), \quad|x| \longrightarrow \infty .
\end{gathered}
$$

It is known that (see [32]) this problem has only the trivial solution: $v\left[h_{0}\right](x) \equiv 0$ in $\mathscr{D}_{0}$. Using the theorem on the jump of the double layer potential [32] and using (3.8), we obtain

$$
\lim _{\substack{x^{1} \rightarrow x \in \Gamma_{0} \\ x^{1} \in \mathscr{D}}} v\left[h_{0}\right]\left(x^{1}\right)-\lim _{\substack{x^{1} \rightarrow x \in \Gamma_{0} \\ x \in \mathscr{D}_{0}}} v\left[h_{0}\right]\left(x^{1}\right)=-h_{0}(x)=0,
$$

so that $h_{0}(x) \equiv 0$ on $\Gamma_{0}$, if $\Gamma_{0} \subset \Gamma$. On the basis of previous results $h_{0}(x) \equiv 0$ on $\Gamma$ in case of both interior and exterior domain $\mathscr{D}$.

Thereby we arrive at the contradiction to the assumption that $h_{0}(x)$ is a nontrivial solution of the homogeneous equation (3.1) (with $g \equiv 0$ ). Thus, the homogeneous equation (3.1) has only the trivial solution. Since Fredholm alternative holds for (3.1), we arrive at the following statement.

LEMMA 3.2. Equation (3.1) is uniquely solvable in $C^{0}(\Gamma)$ for any right-hand side $g(x) \in C^{0}(\Gamma)$.

The inverse operator to $(-1 / 2-A)$ is denoted by $(-1 / 2-A)^{-1}$, then the solution of (3.1) can be expressed in the form

$$
h(x)=\left(\left(-\frac{1}{2}-A\right)^{-1} g\right)(x) .
$$

Let $g(t, x) \in C^{0}\left([0, \infty) ; C^{0}(\Gamma)\right)$. Consider an analogue of (3.1) with respect to the function $h(t, x) \in C^{0}\left([0, \infty) ; C^{0}(\Gamma)\right)$,

$$
-\frac{1}{2} h(t, x)-(A h)(t, x)=g(t, x) .
$$

For any fixed $t$, the operator $(-1 / 2-A)$ is invertible,

$$
h(t, x)=\left(\left(-\frac{1}{2}-A\right)^{-1} g\right)(t, x),
$$

and for any $t, h(t, x) \in C^{0}(\Gamma)$.

Since the operator $(-1 / 2-A)$ is linear and bounded in $C^{0}(\Gamma)$ (see item (2) of Lemma 2.4), then thanks to Banach theorem [31, page 59], the linear operator $(-1 / 2-A)^{-1}$ is also bounded in $C^{0}(\Gamma)$. Therefore, for any $t \geq 0$,

$$
\|h(t, \cdot)\|_{C^{0}(\Gamma)} \leq c_{A}\|g(t, \cdot)\|_{C^{0}(\Gamma)},
$$

where $c_{A}$ is a constant. From the boundedness of the operator $(-1 / 2-A)^{-1}$ it follows its continuity. This means that if

$$
\left\|g(t, \cdot)-g\left(t_{0}, \cdot\right)\right\|_{C^{0}(\Gamma)} \longrightarrow 0, \quad t \longrightarrow t_{0},
$$


then for any functions $h(t, x), h\left(t_{0}, x\right)$, constructed on the basis of formula (3.24), the following relationship holds

$$
\left\|h(t, \cdot)-h\left(t_{0}, \cdot\right)\right\|_{C^{0}(\Gamma)} \longrightarrow 0, \quad t \longrightarrow t_{0} .
$$

Hence $h(t, x) \in C^{0}\left([0, \infty) ; C^{0}(\Gamma)\right)$. Thereby the lemma holds.

LEMmA 3.3. If $g(t, x) \in C^{0}\left([0, \infty) ; C^{0}(\Gamma)\right)$, then (3.23) has the unique solution $h(t, x)$ of class $C^{0}\left([0, \infty) ; C^{0}(\Gamma)\right)$, and this solution obeys estimate (3.25).

4. Solution of the integral equation and the existence theorem. We seek the solution of the integral equation (2.13) in the form of the series [10],

$$
\mu(t, x)=\sum_{m=0}^{\infty} \mu_{m}(t, x)
$$

where the functions $\mu_{m}(t, x) \in C^{0}\left([0, \infty) ; C^{0}(\Gamma)\right)$ are subsequently defined from the equalities, which are obtained when substituting (4.1) into (2.13) formally

$$
\begin{aligned}
-\frac{1}{2} \mu_{0}(t, x)-\left(A \mu_{0}\right)(t, x) & =f(t, x), \\
-\frac{1}{2} \mu_{m}(t, x)-\left(A \mu_{m}\right)(t, x) & =-\left(B \mu_{m-1}\right)(t, x), \quad m=1,2, \ldots . .
\end{aligned}
$$

Since operator $A$ does not depend on $t$, this variable in (4.2) and (4.3) is a parameter.

Since the function $f(t, x) \in C^{0}\left([0, \infty) ; C^{0}(\Gamma)\right)$, then according to Lemma 3.3, equation (4.2) has the unique solution $\mu_{0}(t, x) \in C^{0}\left([0, \infty) ; C^{0}(\Gamma)\right)$, which satisfies the following inequality

$$
\left\|\mu_{0}(t, \cdot)\right\|_{C^{0}(\Gamma)} \leq c_{A}\|f(t, \cdot)\|_{C^{0}(\Gamma)} \leq c_{A}\|f\|_{C^{0}\left([0, t] ; C^{0}(\Gamma)\right)} .
$$

The norm in the Banach space $C^{0}\left([0, T] ; C^{0}(\Gamma)\right)$ is defined by

$$
\|\cdot\|_{C^{0}\left([0, T] ; C^{0}(\Gamma)\right)}=\|\| \cdot\left\|_{C^{0}(\Gamma)}\right\|_{C^{0}[0, T]} \cdot
$$

Substituting $\mu_{0}(t, x)$ found above into the right side of (4.3), we obtain the equation with respect to $\mu_{1}(t, x)$ :

$$
-\frac{1}{2} \mu_{1}(t, x)-\left(A \mu_{1}\right)(t, x)=-\left(B \mu_{0}\right)(t, x) .
$$

Using item (3) of Lemma 2.4, $\left(B \mu_{0}\right)(t, x) \in C^{0}\left([0, \infty) ; C^{0}(\Gamma)\right)$.

According to Lemma 3.3, equation (4.6) has the unique solution

$$
\mu_{1}(t, x) \in C^{0}\left([0, \infty) ; C^{0}(\Gamma)\right)
$$

Besides, on the basis of Lemmas 2.4 and 3.3, the function $\mu_{1}(t, x)$ satisfies the estimate

$$
\begin{aligned}
\left\|\mu_{1}(t, \cdot)\right\|_{C^{0}(\Gamma)} & \leq c_{A}\left\|\left(B \mu_{0}\right)(t, \cdot)\right\|_{C^{0}(\Gamma)} \\
& \leq c_{A} c_{B}\left[\int_{0}^{t}\left\|\mu_{0}(\tau, \cdot)\right\|_{C^{0}(\Gamma)} d \tau+\int_{0}^{t} \int_{0}^{t_{1}}\left\|\mu_{0}(\tau, \cdot)\right\|_{C^{0}(\Gamma)} d \tau d t_{1}\right] .
\end{aligned}
$$


We continue the proof by induction. Let $\mu_{m-1}(t, x) \in C^{0}\left([0, \infty) ; C^{0}(\Gamma)\right)$, then $\mu_{m}(t, x)$ must satisfy (4.3). On the basis of Lemma 2.4,

$$
\left(B \mu_{m-1}\right)(t, x) \in C^{0}\left([0, \infty) ; C^{0}(\Gamma)\right) .
$$

Using Lemma 3.3, equation (4.3) has the unique solution $\mu_{m}(t, x) \in C^{0}([0, \infty)$; $C^{0}(\Gamma)$ ). Using Lemmas 2.4 and 3.3 this solution satisfies the estimate

$$
\begin{aligned}
\left\|\mu_{m}(t, \cdot)\right\|_{C^{0}(\Gamma)} \leq & c_{A} c_{B}\left[\int_{0}^{t}\left\|\mu_{m-1}(\tau, \cdot)\right\|_{C^{0}(\Gamma)} d \tau+\int_{0}^{t} \int_{0}^{t_{1}}\left\|\mu_{m-1}(\tau, \cdot)\right\|_{C^{0}(\Gamma)} d \tau d t_{1}\right] \\
\leq & c_{A}^{2} c_{B}^{2}\left[\int_{0}^{t} \int_{0}^{t_{1}}\left\|\mu_{m-2}(\tau, \cdot)\right\|_{C^{0}(\Gamma)} d \tau d t_{1}\right. \\
& \left.\quad+\int_{0}^{t} \int_{0}^{t_{1}} \int_{0}^{t_{2}} \int_{0}^{t_{3}}\left\|\mu_{m-2}(\tau, \cdot)\right\|_{C^{0}(\Gamma)} d \tau d t_{3} d t_{2} d t_{1}\right] \\
\leq & \quad c_{A}^{m} c_{B}^{m}\left[\int_{0}^{t} \int_{0}^{t_{1}} \cdots \int_{0}^{t_{m-1}}\left\|\mu_{0}(\tau, \cdot)\right\|_{C^{0}(\Gamma)} d \tau d t_{m-1} \cdots d t_{1}\right. \\
& \left.\quad+\int_{0}^{t} \int_{0}^{t_{1}} \cdots \int_{0}^{t_{2 m-1}}\left\|\mu_{0}(\tau, \cdot)\right\|_{C^{0}(\Gamma)} d \tau d t_{2 m-1} \cdots d t_{1}\right] \\
\leq & c_{A}^{m+1} c_{B}^{m}\|f\|_{C^{0}\left([0, t] ; C^{0}(\Gamma)\right)}\left[\int_{0}^{t} \int_{0}^{t_{1}} \cdots \int_{0}^{t_{m-1}} d \tau d t_{m-1} \cdots d t_{1}\right. \\
& \left.+\int_{0}^{t} \int_{0}^{t_{1}} \cdots \int_{0}^{t_{2 m-1}} d \tau d t_{2 m-1} \cdots d t_{1}\right] \\
= & \left.c_{A}\|f\|_{C^{0}\left([0, t] ; C^{0}(\Gamma)\right)} \frac{\left(c_{B} c_{A} t\right)^{m}}{m !}+\frac{\left(\sqrt{c_{B} C_{A}} t\right)^{2 m}}{(2 m) !}\right)
\end{aligned}
$$

where the inequality (4.4) has been used.

Thus, all functions $\mu_{m}(t, x)$, making up series (4.1), are found subsequently. On the basis of Lemma 2.1 each function $\mu_{m}(t, x)$ belongs to $C^{0}([0, \infty) \times \Gamma)$. Besides, each function meets inequality (4.10).

Let us show that series (4.1) converges absolutely and uniformly in $[0, T] \times \Gamma$ for any $T>0$. To prove this we use the Weierstrass theorem [12] and majorize the terms of series (4.1) by terms of the convergent number series. Employing inequality (4.10), we obtain that for any $(t, x) \in[0, T] \times \Gamma$ the following inequality holds

$$
\begin{aligned}
\left|\mu_{m}(t, x)\right| & \leq\left\|\mu_{m}(t, \cdot)\right\|_{C^{0}(\Gamma)} \leq \sup _{t \in[0, T]}\left\|\mu_{m}(t, \cdot)\right\|_{C^{0}(\Gamma)}=\left\|\mu_{m}\right\|_{C^{0}\left([0, T] ; C^{0}(\Gamma)\right)} \\
& \leq c_{A}\left[\frac{\left(c_{A} c_{B} T\right)^{m}}{m !}+\frac{\left(\sqrt{c_{A} C_{B}} T\right)^{2 m}}{(2 m) !}\right] \times\|f\|_{C^{0}\left([0, T] ; C^{0}(\Gamma)\right)} .
\end{aligned}
$$

Thereby any term of the series (4.1) is majorized on the set $[0, T] \times \Gamma$ by the term of the number series

$$
c_{A}\|f\|_{C^{0}\left([0, T] ; C^{0}(\Gamma)\right)}\left[\frac{\left(c_{A} c_{B} T\right)^{m}}{m !}+\frac{\left(\sqrt{c_{A} c_{B}} T\right)^{2 m}}{(2 m) !}\right],
$$


which converges and its sum is equal to

$$
c_{A}\|f\|_{C^{0}\left([0, T] ; C^{0}(\Gamma)\right)}\left[\exp \left(c_{A} c_{B} T\right)+\operatorname{ch}\left(\sqrt{c_{A} C_{B}} T\right)\right] .
$$

Consequently, using the Weierstrass theorem, series (4.1) converges absolutely and uniformly on the set $[0, T] \times \Gamma$ for any $T>0$. These arguments and continuity of the terms of the series prove that the series can be integrated term-by-term when substituting this series in (2.13). Moreover, the integrated series is also absolutely and uniformly convergent [12] on $[0, T] \times \Gamma$ for any $T>0$, and so its terms can be summed in an arbitrary order. Using the subsequence of equalities (4.2) and (4.3), equation (2.13) becomes an identity. Thereby the function $\mu(t, x)$, defined as a sum of series (4.1), obeys (2.13), because all formal operations used in the substitution of (4.1) into (2.13) are justified.

According to the theorem on continuity of the sum of a series [12], from uniform convergence of series $(4.1)$ in $[0, T] \times \Gamma$ for any $T>0$, and from continuity of its terms in $[0, \infty) \times \Gamma$ it follows that $\mu(t, x) \in C^{0}([0, \infty) \times \Gamma)$. By Lemma $2.1, \mu(t, x) \in$ $C^{0}\left([0, \infty) ; C^{0}(\Gamma)\right)$. Thus, the desired solution of (2.13) has been constructed.

THEOREM 4.1. For any function $f(t, x) \in C^{0}\left([0, \infty) ; C^{0}(\Gamma)\right)$ equation (2.13) has a solution $\mu(t, x) \in C^{0}\left([0, \infty) ; C^{0}(\Gamma)\right)$.

Note that a function $f(t, x)=\left[f_{0}(t, x)\right]_{t t} \in C^{0}\left([0, \infty) ; C^{0}(\Gamma)\right)$ in a right-hand side of the integral equation (2.13) corresponds to an arbitrary function $f_{0}(t, x) \in C_{0}^{2}([0, \infty)$; $\left.C^{0}(\Gamma)\right)$ in the boundary condition (1.3) of the problem $\mathscr{K}$.

Theorem 4.1 ensures the existence of the solution $\mu(t, x)$ of the integral equation (2.13) in the required class of smoothness. Hence, the potential (2.12), constructed for this function $\mu(t, x)$, satisfies the boundary condition of the problem $\mathscr{K}$. Besides, according to Lemma 2.3, the potential (2.12) constructed in such a way belongs to the smoothness class $G$ and satisfies all other conditions of the problem $\mathscr{K}$. We arrive at the following theorem.

THEOREM 4.2. For any function $f_{0}(t, x) \in C_{0}^{2}\left([0, \infty) ; C^{0}(\Gamma)\right)$ the classical solution of the problem $\mathscr{K}$ exists and is given by formula (2.12), where the density $\mu(t, x) \in$ $C^{0}\left([0, \infty) ; C^{0}(\Gamma)\right)$ is the solution of the integral equation (2.13), which is always solvable.

One can show that if $f_{0}(t, x) \in C_{0}^{2}\left([0, \infty) ; C^{1, \lambda}(\Gamma)\right), \lambda \in(0,1]$, then the solution of the problem $\mathscr{K}$ constructed in Theorem 4.2 belongs to the smoothness class $G_{1}$. In this case, the solution of problem $\mathscr{K}$ is unique, by Theorem 1.5.

ACKNowledgement. The author is thankful to Professor K. Kajitani for the opportunity to carry out research in Japan. Also, the support of the University of Tsukuba is gratefully acknowledged.

\section{REFERENCES}

[1] L. M. Brekhovskikh and V. Goncharov, Mechanics of Continua and Wave Dynamics, 2nd ed., Springer Series on Wave Phenomena, vol. 1, Springer-Verlag, Berlin, 1994. MR 96a:73002. Zbl 791.73001.

[2] D. L. Colton and R. Kress, Integral Equation Methods in Scattering Theory, Pure and 
Applied Mathematics, John Wiley \& Sons, New York, 1983, A Wiley-Interscience Publication. MR 85d:35001. Zbl 522.35001.

[3] L. Debnath (ed.), Nonlinear Waves, Cambridge Monographs on Mechanics and Applied Mathematics, Cambridge University Press, Cambridge, 1983, Papers presented at the conference on nonlinear waves and integrable systems held at East Carolina University, North Carolina, June 1982. MR 85d:76001. Zbl 536.00016.

[4] _ Nonlinear Water Waves, Academic Press, Massachusetts, 1994. MR 95c:76011. Zbl 793.76001.

[5] L. Debnath (ed.), Advances in Nonlinear Waves. Vol. I, Research Notes in Mathematics, vol. 95, Pitman (Advanced Publishing Program), London, 1984. MR 87a:76002a. Zbl 535.00028 .

[6] _ Advances in Nonlinear Waves. Vol. II, Research Notes in Mathematics, vol. 111, Pitman (Advanced Publishing Program), London, 1985. MR 87a:76002b. Zbl 563.00022 .

[7] Y. V. Egorov, Linear Differential Equations of Principal Type, Contemporary Soviet Mathematics, Consultants Bureau, New York, 1986, translated from the Russian by Dang Prem Kumar. MR 88c:35003. Zbl 669.35001.

[8] S. A. Gabov and P. V. Shevtsov, Basic boundary value problems for the equation of oscillations of a stratified fluid, Soviet Math. Dokl. 27 (1983), 238-241 (Russian), [translation from Dokl. Akad. Nauk SSSR 268 (1983), 1293-1296. MR 85a:35081]. Zbl 546.76128.

[9] _ On a differential equation of the type of Sobolev's equation, Soviet Math. Dokl. 29 (1984), 411-414 (Russian), [translation from Dokl. Akad. Nauk SSSR 276 (1984), 14-17. MR 86a:35134]. Zbl 597.35084.

[10] S. A. Gabov and A. G. Sveshnikov, Linejnye Zadachi Teorii Nestatsionarnykh Vnutrennikh Voln [Linear Problems of the Theory of Non-stationary Internal Waves], "Nauka", Moscow, 1990 (Russian), with an English summary. MR 92i:76015. Zbl 713.76003.

[11] H. P. Greenspan, The Theory of Rotating Fluids, Cambridge University Press, New York, 1980, reprint of the 1968 original. Cambridge Monographs on Mechanics and Applied Mathematics. MR 83m:76077. Zbl 443.76090.

[12] V. Il'in and E. Pozniak, Fundamentos del Análisis Matemático. 2 [Foundations of Mathematical Analysis. 2], Editorial Mir, Moscow, 1991 (Spanish), translated from the Russian by M. Andrianova and K. Medkov. An early edition of the Russian original of part 1 has also been reviewed [“Nauka," Moscow, 1971 MR 50\#2404]. MR 92g:00002b.

[13] K. Kajitani and T. Nishitani, The Hyperbolic Cauchy Problem, Lecture Notes in Mathematics, vol. 1505, Springer-Verlag, Berlin, 1991. MR 94f:35074. Zbl 762.35002.

[14] B. V. Kapitonov, Potential theory for the equation of small oscillations of a rotating fluid, Math. USSR-Sb. 37 (1980), 559-579. Zbl 452.35010.

[15] P. A. Krutitskiĭ, Explicit solution of the Dirichlet problem for an equation of composite type in a multiply connected domain, Russian Acad. Sci. Dokl. Math. 46 (1993), no. 1, 63-69 (Russian), [translation from Dokl. Akad. Nauk, Ross. Akad. Nauk 325 (1992), no. 3, 428-433. MR 94d:35117]. Zbl 806.35124.

[16] _ Reduction of the second initial-boundary value problem for the equation of gravitational-gyroscopic waves to a uniquely solvable integral equation, Differential Equations 32 (1996), no. 10, 1383-1392 (Russian), [translation from Differ. Uravn. 32 (1996), no. 10, 1386-1395. MR 98m:76045]. Zbl 896.35089.

[17] _ The second initial-boundary value problem for an equation of gravitationalgyroscopic waves in an exterior domain, Math. Notes 60 (1996), no. 1, 29-41, [translation from Mat. Zametki 60 (1996), no. 1, 40-57. MR 98c:76021]. Zbl 898.35064.

[18] _ The first initial-boundary value problem for the gravity-inertia wave equation in a multiply connected domain, Comput. Math. Math. Phys. 37 (1997), no. 1, 113-123 (Russian), [translation from Zh. Vychisl. Mat. Mat. Fiz. 37 (1997), no. 1, 117-128. MR 98a:76016]. Zbl 946.83014. 
[19] _ An initial-boundary value problem for the pseudo-hyperbolic equation of gravitygyroscopic waves, J. Math. Kyoto Univ. 37 (1997), no. 2, 343-365. MR 98g:76016. Zbl 892.35109.

[20] Exterior initial-boundary value problem for an evolution equation in a multiply connected domain, Differential Equations 34 (1998), no. 12, 1629-1638, [translation from Differ. Uravn. 34 (1998), no. 12, 1624-1633. CMP 1723 146.]. Zbl 953.35105.

[21] H. Lamb, Hydrodynamics, Cambridge Mathematical Library, Cambridge University Press, Cambridge, 1993, reprint of the 1932 sixth edition. With a foreword by R. A. Caflisch [Russel E. Caflisch]. MR 96f:76001. Zbl 828.01012.

[22] P. H. le Blond and L. A. Mysak, Waves in the Ocean, Elsevier, Amsterdam, 1978.

[23] C. Miranda, Partial Differential Equations of Elliptic Type, 2nd ed., translated from the Italian by Zane C. Motteler. Ergebnisse der Mathematik und ihrer Grenzgebiete, vol. 2, Springer-Verlag, New York, 1970. MR 44\#1924. Zbl 198.14101.

[24] S. Mizohata, The Theory of Partial Differential Equations, Cambridge University Press, New York, 1973, Translated from the Japanese by Katsumi Miyahara. MR 58\#29033. Zbl 263.35001.

[25] _ On the Cauchy Problem, Notes and Reports in Mathematics in Science and Engineering, vol. 3, Academic Press, Florida; Science Press, Beijing, 1985. MR 89a:35007. Zbl 616.35002.

[26] M. H. Protter and H. F. Weinberger, Maximum Principles in Differential Equations, SpringerVerlag, New York, 1984, Corrected reprint of the 1967 original. MR 86f:35034. Zbl 549.35002.

[27] S. L. Sobolev, On a new problem of mathematical physics, Izv. Akad. Nauk SSSR Ser. Mat. 18 (1954), 3-50 (Russian). MR 16:1029d. Zbl 055.08401.

[28] _ Sur une classe des problèmes de physique mathématique, Simpos. Internaz. Appl. Anal. Fis. Mat. (Cagliari-Sassari, 1964) (Rome), Edizioni Cremonese, 1965, pp. $192-$ 208 (French). MR 36\#6783. Zbl 142.22602.

[29] _ Izbrannye Voprosy Teorii Funktsional'nykh Prostranstv i Obobshchennykh Funktsij [Selected Questions of the Theory of Functional Spaces and Generalized Functions], with an English summary. Edited and with a preface by S. V. Uspenskii, "Nauka", Moscow, 1989 (Russian). MR 90m:46060. Zbl 667.46025.

[30] S. V. Uspenskiı̌, G. V. Demidenko, and V. G. Perepelkin, Teoremy Vlozheniya $i$ Prilozheniya k Differentsial'nym Uravneniyam [Imbedding Theorems and Applications to Differential Equations], "Nauka” Sibirsk. Otdel., Novosibirsk, 1984 (Russian). MR 86i:46038. Zbl 575.46030.

[31] N. J. Vilenkin, E. A. Gorin, A. G. Kostjučenko, M. A. Krasnosel’skiı̌, S. G. Kreǐn, V. P. Maslov, B. S. Mitjagin, J. I. Petunin, J. B. Rutickiǐ, V. I. Sobolev, V. J. Stecenko, L. D. Faddeev, and È. S. Citlanadze, Funktsionalyanyı̆ Analiz [Functional Analysis], Edited by S. G. Krěin, Izdat. "Nauka”, Moscow, 1964 (Russian). MR 32\#1532.

[32] V. S. Vladimirov, Equations of Mathematical Physics, translated from the Russian by Audrey Littlewood. Edited by Alan Jeffrey. Pure and Applied Mathematics, vol. 3, Marcel Dekker, New York, 1971. MR 42\#3394. Zbl 207.09101.

Pavel A. KrutitskiI: Department of Mathematics, Faculty of Physics, Moscow State UNIVERSITY, MOSCOW 117234, RUSSIA

E-mail address: krutitsk@math.phys.msu.su 


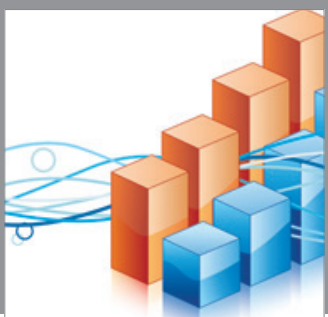

Advances in

Operations Research

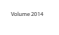

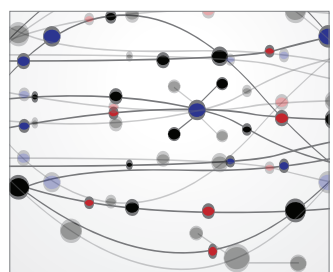

\section{The Scientific} World Journal
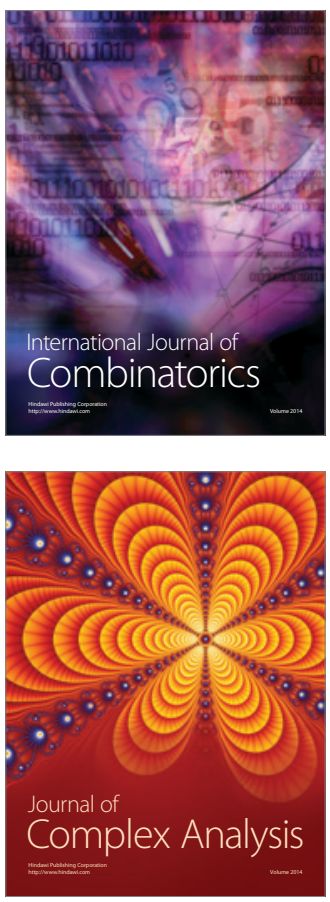

International Journal of

Mathematics and

Mathematical

Sciences
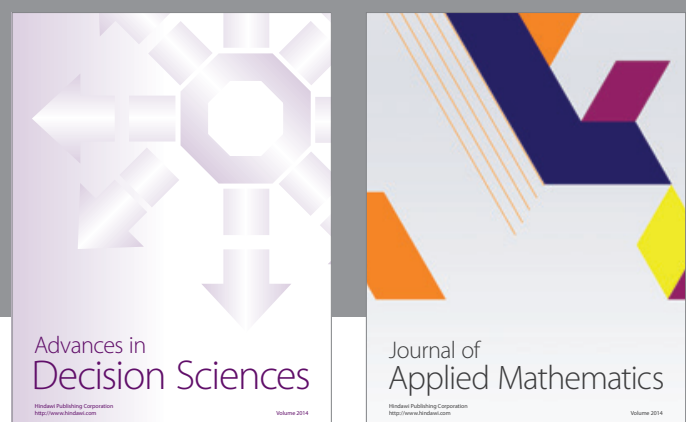

Journal of

Applied Mathematics
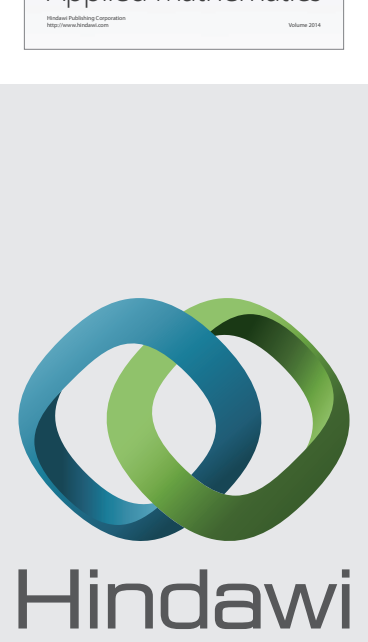

Submit your manuscripts at http://www.hindawi.com
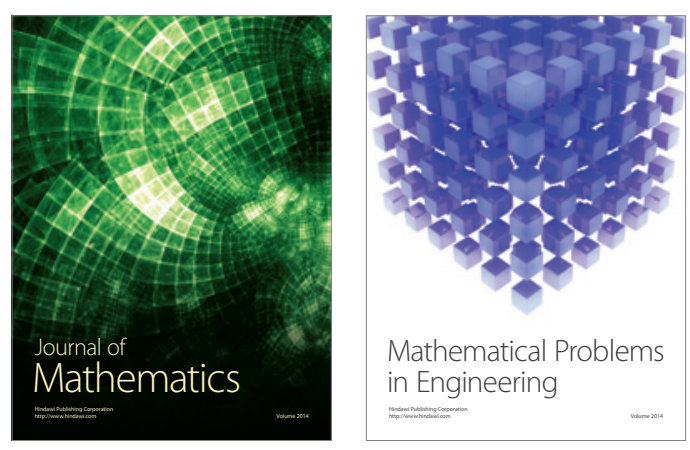

Mathematical Problems in Engineering
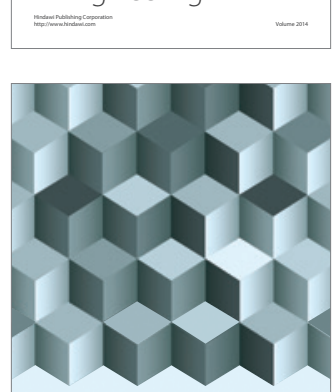

Journal of

Function Spaces
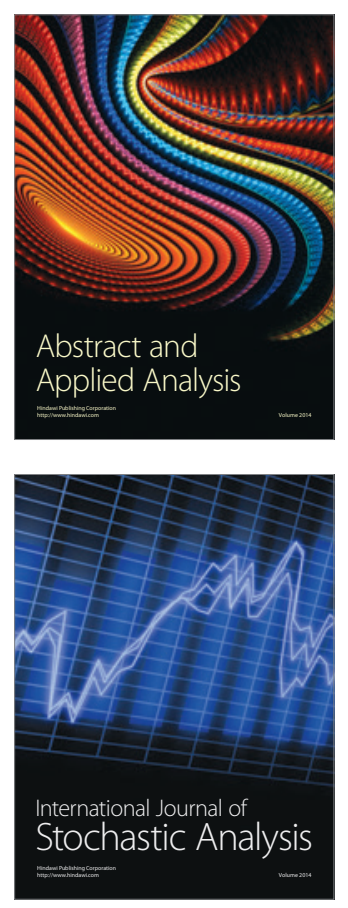

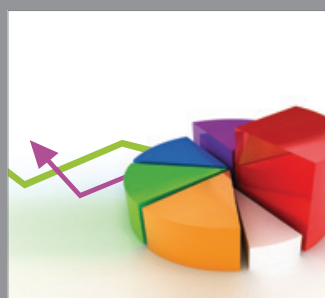

ournal of

Probability and Statistics

Promensencen
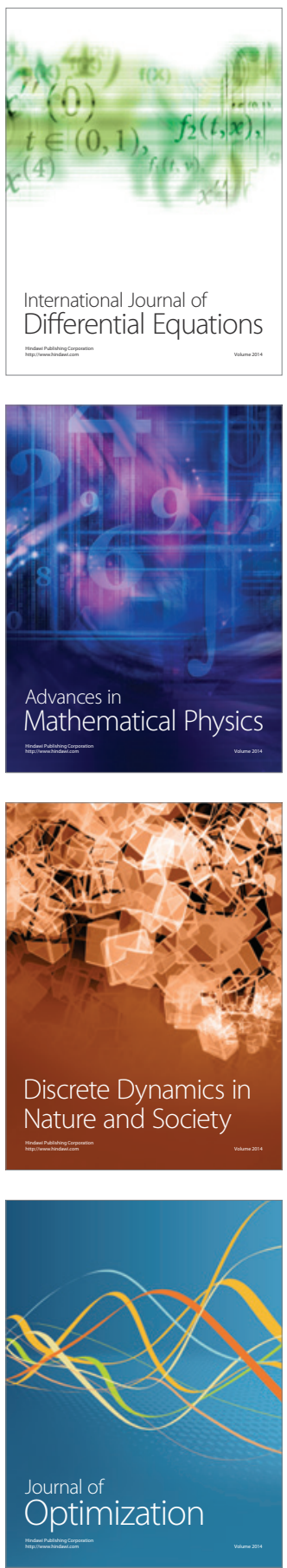